\title{
FSH dose to stimulate different patient' ages: when less is more
}

\author{
Edson Borges Jr. ${ }^{1,2}$, Bianca F Zanetti ${ }^{1,2}$, Amanda S Setti ${ }^{1,2}$, Daniela PAF Braga ${ }^{1,2,3}$, Rita de Cássia S Figueira ${ }^{1}$, \\ Assumpto Iaconelli Jr. ${ }^{1,2}$
}

${ }^{1}$ Fertility - Medical Group, São Paulo, SP - Brazil

2Instituto Sapientiae - Centro de Estudos e Pesquisa em Reprodução Humana Assistida, São Paulo, SP - Brazil ${ }^{3}$ Disciplina de Urologia, Área de Reprodução Humana, Departamento de Cirurgia, Universidade Federal de São Paulo. - UNIFESP

\begin{abstract}
Objective: To determine the effect of FSH doses on intracytoplasmic sperm injection (ICSI) outcomes according to the age of the patient.

Methods: Patients undergoing controlled ovarian stimulation (COS) for ICSI cycles in a university-affiliated in vitro fertilization center were split into age groups: $\leq 35$ y.o. $(n=1523) ;>35$ and $\leq 38$ y.o. $(n=652) ;>38$ and $\leq 40$ y.o. $(n=332)$; and $>40$ y.o. $(n=370)$. The effect of FSH dose on COS, laboratorial and clinical outomes was determined by linear regression models.

Results: The FSH dose didn't affect the ovarian response in terms of total number of follicles, retrieved oocytes and mature oocytes within the age groups, but we found that the lower the age, the lower the FSH dose needed per oocyte retrieved. In the group of patients $\leq 35$ y.o., we also found a positive effect of the FSH dose on oocyte yield. Despite that, for patients $\leq 38 \mathrm{y} .0$. there was a negative effect of the FSH dose on embryo quality and blastocyst formation rate, and an increase in the cycle's cancelation rate. In patients $\geq 39$ y.o., there were no effects of the FSH doses on the analysed variables.

Conclusions: Ovarian stimulation with high doses of $\mathrm{FSH}$ is not recommended in younger women ( $\leq 38$ y.o.), once we found a decrease in embryo quality and an increase in cycle's cancelation rate. Mild ovarian stimulation protocols may be more appropriate; however, it may not be applicable for women in advanced age, since a higher FSH dose is needed for oocyte retrieval in these patients.
\end{abstract}

Keywords: FSH, controlled ovarian stimulation, female age, oocyte yield, IVF

\section{INTRODUCTION}

In vitro fertilization success rates have been remarkably improved since the procedure was first established for clinical use, with the first successful birth in 1978 (Steptoe \& Edwards, 1978). Nevertheless, its efficiency, measured as live birth rate, is usually well below $50 \%$. Many couples must undergo several Assisted Reproductive Technology (ART) cycles before they succeed in becoming parents. These multiple trials increase emotional and financial costs, and bring additional risks for women's health (Patrizio \& Sakkas, 2009; Seli et al., 2010).

The success of in vitro fertilization (IVF) depends on the number and quality of retrieved oocytes and embryos obtained. For that, we employ complex and costly controlled ovarian stimulation (COS) protocols to generate multiple embryos (Fauser et al., 2005). After ovarian stimulation and IVF, the best quality embryos are selected for transfer into the uterine cavity.

Conventional COS regimens routinely use a gonadotrophin-releasing hormone $(\mathrm{GnRH})$ agonist or antagonist to prevent a premature luteinizing hormone (LH) rise, and high doses of exogenous FSH are administered to induce multiple follicle growth (Sunkara et al., 2011; Steward et al., 2014). This traditional COS cause considerable discomfort to the patients, and has important short-term complications, including ovarian hyperstimulation syndrome (OHSS), and a high incidence of multiple pregnancies (Pandian et al., 2009; Setti \& Bulletti, 2011). These negative aspects lead to a high rate of drop-outs (Verberg et al., 2008), and increased costs (Goverde et al., 2000). On the other side, a significant proportion of patients show a low or poor response to the classical approach (Bosch \& Ezcurra, 2011).

In addition, embryo implantation does not depend exclusively on proper embryo development. It also involves having a receptive endometrium, and a proper synergy with embryos (Dominguez et al., 2003). Growing evidence in the literature shows that COS, with its supraphysiological hormone levels, may decrease endometrial receptivity (Devroey et al., 2004; Shapiro et al., 2011) and embryo quality (Sunkara et al., 2015), increasing embryo implantation failure.

An adequate COS should lead to not only an increased number of retrieved oocytes but also to high quality oocytes and embryos with increased implantation potential, combined with maternal receptive endometrium (Sunkara et al., 2016). However, the response to COS is age dependent and sometimes its unpredictability is a barrier to the implementation of individualized COS protocols (Aanesen et al., 2010; Polyzos et al., 2012).

The determination of the best gonadotrophin dose to be applied for different kinds of patients is extremely important; therefore, the goal of the present study was to establish the effect of different recombinant FSH doses on oocytes and embryo quality, according to patient age.

\section{METHODS}

\section{Study design}

This retrospective cohort study included 12,334 embryos obtained from 2,877 patients undergoing COS, and intracytoplasmic sperm injection (ICSI) cycles. Cycles were performed in a private university-affiliated IVF center, between January/2010 and December/2016. The patients were split into different age groups: $\leq 35$ years old $(n=1523) ;>35$ and $\leq 38$ years old $(n=652) ;>38$ and $\leq 40$ years old $(n=332)$; and $>40$ years old $(n=370)$.

The effect of the FSH dose on (i) the number of follicles, (ii) the number of retrieved oocytes; (iii) oocyte yield (number of retrieved oocytes/number of follicles); (iv) number of mature oocytes, (v) mature oocyte rate, (vi) fertilization rate, (vii) embryo quality at cleavage stage, (viii) blastocyst formation rate, (ix) endometrial thickness, (x) cycle's cancelation rate, (xi) implantation rate, (xii) 
pregnancy rate, and (xii) miscarriage rate, was evaluated on the different age intervals.

The oocytes were evaluated immediately before sperm injection, and the embryos were evaluated $16-18 \mathrm{~h}$ postICSI and on days three and five of development.

For embryo quality evaluation, the embryos were classified as high or low quality on days three and five.

The implantation rate was defined as the number of gestational sacs divided by the number of embryos transferred per patient. Clinical pregnancy was defined as the presence of a gestational sac with a heartbeat detected by ultrasound 4-6 weeks after embryo transfer.

Written informed consent, in which patients agreed to share the outcomes of their cycles for research purposes, were obtained, and the local institutional review board approved the study.

\section{Controlled ovarian stimulation}

Controlled ovarian stimulation was achieved using a daily dose of recombinant FSH ( $r-F S H$, Gonal- $F^{\circledR}$, Merck $\mathrm{KGaA}$, Darmstadt, Germany) beginning on day three of the cycle. Pituitary blockage was performed using a GnRH antagonist (GnRHa, Cetrotide ${ }^{\circledR}$; Merck KGaA, Darmstadt, Germany), beginning when at least one follicle $\geq 14 \mathrm{~mm}$ was visualized.

Follicular growth was monitored using transvaginal ultrasound, beginning on day four of the gonadotropin administration. When adequate follicular growth, and serum E2 levels were noticed, recombinant hCG ( $r$-hCG, Ovidrel ${ }^{\circledR}$, Merck KGaA, Darmstadt, Germany), was administered to trigger the final follicular maturation. The oocytes were collected 35 hours after hCG administration through transvaginal ultrasound ovum pick-up.

\section{Preparation of oocytes}

Retrieved oocytes were maintained in culture medium (Global ${ }^{\circledR}$ for fertilization, LifeGlobal, Connecticut, USA) supplemented with $10 \%$ protein supplement (LGPS, LifeGlobal, Connecticut, USA) and covered with paraffin oil (Paraffin oil P.G., LifeGlobal, Connecticut, USA) for two to three hours before removing the cumulus cells. The surrounding cumulus cells were removed after exposure to a HEPES-buffered medium containing hyaluronidase (80 IU/mL, LifeGlobal, Connecticut, USA). The remaining cumulus cells were mechanically removed by gently pipetting with a hand-drawn Pasteur pipette (Humagen Fertility Diagnostics, Charlottesville, USA).

The oocyte morphology was assessed immediately before sperm injection (4 hours after retrieval) using an inverted Nikon Diaphot microscope (Eclipse TE 300; Nikon ${ }^{\circledR}$, Tokyo, Japan) with a Hoffmann modulation contrast system under 400X magnification. Oocytes that released the first polar body were considered mature and used for ICSI.

\section{Intracytoplasmic sperm injection}

Intracytoplasmic sperm injection was performed in a micro-injection dish prepared with $4-\mu \mathrm{L}$ droplets of buffered medium (Global ${ }^{\circledR}$ W/HEPES, LifeGlobal, Connecticut, USA) and covered with paraffin oil on the heated stage in an inverted microscope $\left(37.0 \pm 0.5^{\circ} \mathrm{C}\right)$. Approximately 16 hours after ICSI, fertilization was confirmed by the presence of two pronuclei and the extrusion of the second polar body. Embryos were maintained in a 50- $\mu \mathrm{L}$ drop of culture medium (Global ${ }^{\circledR}$, LifeGlobal, Connecticut, USA), supplemented with $10 \%$ protein supplement and covered with paraffin oil in a humidified atmosphere, under $6 \% \mathrm{CO}_{2}$ at $37^{\circ} \mathrm{C}$ for five days.

\section{Embryo morphology evaluation}

Embryo morphology was assessed $16-18 \mathrm{~h}$ post-ICSI and on the mornings of days two, three, and five using an inverted Nikon Diaphot microscope (Eclipse TE 300; Nikon, Tokyo, Japan) with a Hoffmann modulation contrast system under $400 \mathrm{X}$ magnification.

To evaluate the cleavage-stage morphology, the following parameters were recorded: the number of blastomeres, the percentage of fragmentation, the variation in blastomere symmetry, the presence of multinucleation and the defects in the zona pellucida and cytoplasm. The high-quality cleavage-stage embryos were defined as those with the following characteristics: 4 cells on day two, or 8-10 cells on day three, $<15 \%$ fragmentation, symmetric blastomeres, the absence of multinucleation, colorless cytoplasm with moderate granulation and no inclusions, the absence of perivitelline space granularity and the absence of zona pellucida dysmorphism. Embryos lacking any of these characteristics were of low quality.

The size and compactness of the ICM and the cohesiveness and number of TE cells were recorded to evaluate the blastocyst-stage morphology. The embryos received a numerical score from one to six, based on their degree of expansion and hatching status as follows: 1 , an early blastocyst with a blastocele that was less than half of the volume of the embryo; 2 , a blastocyst with a blastocele that was greater than half of the embryo volume; 3, a full blastocyst with a blastocele that filled the embryo; 4, an expanded blastocyst; 5, a hatching blastocyst; and 6, a hatched blastocyst. The ICM of full, expanded, hatching, and hatched blastocysts were classified as either high quality (tightly packed with many cells) or low quality (loosely grouped with several or few cells). Similarly, TEs were classified as either being of high quality (many cells forming a cohesive epithelium) or low quality (few cells forming a loose epithelium or very few cells).

Embryo transfer was performed on the fifth day of development.

\section{Clinical Follow-up}

A pregnancy test was performed 12 days after embryo transfer. All women with a positive test were submitted to a transvaginal ultrasound scan 2 weeks after the positive test. Clinical pregnancy was diagnosed when the fetal heartbeat was detected. Pregnancy rates were calculated per transfer. Miscarriage was defined as pregnancy loss before 20 weeks.

\section{Statistical analyses}

The FSH dose effect on the number of follicles, number of retrieved oocytes, oocyte yield, number of mature oocytes, mature oocyte rate, fertilization rate, embryo quality at cleavage stage, blastocyst formation rate, endometrial thickness, cycle's cancelation rate, implantation rate, pregnancy rate, and miscarriage rate at different age intervals were evaluated by linear regression on the different age intervals ( $\leq 35$ years old, $>35$ and $\leq 38$ years old, $>38$ and $\leq 40$ years old and $>40$ years old). The results are expressed as regression coefficients (RC) and $p$ value.

The FSH/oocyte ratio was calculed as FSH total dose (UI) per number of retrived oocytes. The effects of the age groups on this variable were determined by One-way ANOVA. The results are expressed as mean and $p$ value.

All the statistics analyses were performed at the IBM SPSS 20 Software. 


\section{RESULTS}

A total of 12,334 embryos obtained from 2877 patients undergoing the first ICSI cycles were included in the analysis. The descriptive analysis of the patients' demographics and COS outcomes is shown in Table 1. Laboratorial and clinical outcomes are described in Table 2.

The FSH dose effect was evaluated though linear regression models in different age groups (Tables 3, 4 and 5). The FSH dose didn't affect the ovarian response to $\operatorname{COS}$ (i.e. aspirated follicles, retrieved oocytes, and mature oocytes); however, a positive effect on oocyte yield (number of retrieved oocytes/number of follicles) in the patients $\leq 35$ years old could be seen. Despite that, we found a negative effect of FSH on cleavage stage embryo quality and blastocyst formation rate, for patients $\leq 38$ years old (both groups: $\leq 35$ and $36-38$ years old). In these groups of patients, the cycle's cancelation rate was positivelly affected by the FSH dose. When analysing the cause of cancellation, we found that the main cause was OHSS risk, $89 \%$ and $82.7 \%$, for patients $\leq 35$ an $36-38$ years old, respectively.

In patients $\geq 39$ years old, there were no effects of the $\mathrm{FSH}$ doses on the analysed variables.

The FSH/oocyte ratio (calculed as FSH total dose (UI)/ number of retrived oocytes) was significantly higher over the years (Table 6), which means that a higher FSH dose is need for oocyte retrieval in older women (Figure 1).

\section{DISCUSSION}

An optimal response to COS is crucial for the success of IVF. Either too low or too high ovarian responses to gonadotrophin stimulation are associated with increased cycle's cancellation rates and lower pregnancy rates. Previous reports have suggested an optimal range of oocytes below and above which outcomes are compromised (van der Gaast et al., 2006; Sunkara et al., 2011). However, the ideal dose of FSH for COS which results in optimal oocyte recovery and proper embryo development is still uncertain. This depends on many variables, but the patient's age seems to be the principal factor that affects ovary response to stimulation.

For the present study, the FSH dose effects on laboratorial and clinical ICSI outcomes were evaluated through linear regression models in different age groups. Intriguingly, no significant effect of $\mathrm{FSH}$ dose on the ovary response to $\mathrm{COS}$ (i.e. aspirated follicles, retrieved oocytes and mature oocytes), could be noted. However, for younger patients ( $\leq 35$ years old) we found a direct correlation between the FSH dose and the oocyte yield (oocyte retrieval rate).

Concerning the embryo development, for patients $\leq 38$ years old (both groups: $\leq 35$ and $36-38$ years old), the increased FSH dose had a negative impact on both cleavage stage embryo quality rate and blastocyst formation rate. Moreover an effect on cycles cancelation risk was noted, in which the higher the dose of FSH, the greater the chance of cancellation.

During COS, the supraphysiological doses of gonadotrophins maintain FSH and LH levels above a critical threshold needed to stimulate the development of multiple follicles, enabling the retrieval of many oocytes in a single IVF cycle (Fatemi et al., 2012).

Conventional ovarian stimulation protocols used to maximize the number of collected oocytes and therefore yielding more embryos, has been associated with good clinical outcomes, in the last decades. However, high doses of gonadotrophins may lead to increased patient discomfort and higher costs (Bodri et al., 2008; Verberg et al., 2008; Steward et al., 2014). Moreover, concerns on the embryo developmental potential have been raised.

Although previous reports have demonstrated that higher doses of gonadotrophins used in conventional stimulation protocols are not likely to have a negative effect on embryo quality (Kok et al., 2006) or embryo aneuploidy incidence (Ata et al., 2012; Labarta et al., 2012), other have demonstrated the opposite (Ziebe et al., 2007; Haaf et al., 2009; Braga et al., 2012), which corroborates with our findings. In the present study, no significant effect on pregnancy was observed, but other studies suggested that the increase in gonadotrophins is also associated with lower pregnancy rates (Jenkins et al., 1991; Stadtmauer et al., 1994; Shulman et al., 1995).

In recent years, mild stimulation protocols have risen in popularity. Typically, these protocols use lower doses of gonadotrophins and/or shorter stimulation protocols, aiming to retrieve less than eight oocytes (Fauser et al. 2010). Even though some studies have shown that the pregnancy rate per cycle is lower, milder stimulation may result in the retrieval of good quality oocytes, which, thereafter, may result in high quality embryos with increased implantation potential (Hunault et al., 2002; Hohmann et al., 2003; Baart et al., 2007).

In our study, an increased rate of cycle's cancelation was correlated with higher FSH doses in younger patients.

Table 1. Descriptive analysis of patient demographics and COS outcomes.

\begin{tabular}{|c|c|c|c|c|c|c|c|c|c|c|}
\hline \multirow[t]{2}{*}{ Variable } & \multicolumn{2}{|c|}{ General } & \multicolumn{2}{|c|}{$\leq 35$ years old } & \multicolumn{2}{|c|}{$\begin{array}{c}36-38 \text { years } \\
\text { old }\end{array}$} & \multicolumn{2}{|c|}{$\begin{array}{c}\text { 39-40 years } \\
\text { old } \\
\end{array}$} & \multicolumn{2}{|c|}{$>40$ years old } \\
\hline & Mean & SD & Mean & SD & Mean & SD & Mean & SD & Mean & SD \\
\hline \multicolumn{11}{|l|}{ Patient's demographics } \\
\hline Maternal age $(y)$ & 35.1 & 4.5 & 31.7 & 2.8 & 36.9 & 0.8 & 39.4 & 0.5 & 42.6 & 1.8 \\
\hline Paternal age $(y)$ & 38.0 & 6.6 & 35.7 & 5.9 & 39.3 & 6.1 & 41.3 & 6.0 & 43.0 & 6.7 \\
\hline Total FSH administred (IU) & 2318.8 & 607.9 & 2185.9 & 580.2 & 2442.7 & 574.5 & 2508.9 & 621.0 & 2476.9 & 637.4 \\
\hline \multicolumn{11}{|l|}{ cos outcomes } \\
\hline Aspirated follicles (n) & 16.5 & 12.1 & 15.0 & 9.8 & 14.6 & 10.7 & 11.0 & 8.0 & 8.0 & 6.8 \\
\hline Retrieved oocytes (n) & 11.8 & 9.2 & 14.9 & 19.8 & 10.2 & 8.0 & 7.7 & 6.2 & 5.6 & 5.3 \\
\hline Oocyte Yield (\%) & 70.3 & 22.4 & 72.5 & 19.8 & 68.5 & 22.7 & 66.9 & 25.1 & 67.5 & 28.2 \\
\hline Mature oocytes MII (n) & 8.9 & 7.0 & 11.2 & 7.5 & 7.7 & 6.0 & 6.0 & 4.8 & 4.3 & 4.0 \\
\hline Mature oocytes rate $(\%)$ & 74.5 & 21.0 & 75.1 & 18.5 & 71.4 & 22.4 & 74.5 & 22.8 & 72.8 & 26.6 \\
\hline
\end{tabular}

Note: COS: controlled ovarian stimulation; FSH: follicle-stimulated hormone; SD: standard deviation. 


\begin{tabular}{|c|c|c|c|c|c|c|c|c|c|c|}
\hline \multirow[t]{2}{*}{ Variable } & \multicolumn{2}{|c|}{ General } & \multicolumn{2}{|c|}{$\leq 35$ years old } & \multicolumn{2}{|c|}{$\begin{array}{c}36-38 \text { years } \\
\text { old }\end{array}$} & \multicolumn{2}{|c|}{$\begin{array}{c}39-40 \text { years } \\
\text { old }\end{array}$} & \multicolumn{2}{|c|}{$>40$ years old } \\
\hline & Mean & SD & Mean & SD & Mean & SD & Mean & SD & Mean & SD \\
\hline \multicolumn{11}{|l|}{ Laboratory outcomes } \\
\hline Fertilization rate $(\%)$ & 83.3 & 19.5 & 86.1 & 17.3 & 83.2 & 19.9 & 81.9 & 23.4 & 82.1 & 23.9 \\
\hline High quality D3 embryos (n) & 3.1 & 2.9 & 3.8 & 3.2 & 2.8 & 2.8 & 2.2 & 2.3 & 1.6 & 1.7 \\
\hline High quality Embryo rate (\%) & 45.8 & 31.1 & 48.1 & 28.6 & 42.7 & 32.4 & 42.0 & 32.8 & 45.4 & 37.1 \\
\hline Blastocyst formation rate $(\%)$ & 41.1 & 28.4 & 44.3 & 27.9 & 36.6 & 28.1 & 41.3 & 29.7 & 27.5 & 26.9 \\
\hline Transferred embryos ( $n$ ) & 1.5 & 0.6 & 1.6 & 0.6 & 1.5 & 0.7 & 1.5 & 0.7 & 1.4 & 0.7 \\
\hline Endometrium (mm) & 11.0 & 7.0 & 11.1 & 5.7 & 10.9 & 6.6 & 10.9 & 2.2 & 11.3 & 13.6 \\
\hline \multicolumn{11}{|l|}{ Clinical outcomes } \\
\hline Cancelation rate $(\%)$ & \multicolumn{2}{|c|}{22.4} & \multicolumn{2}{|c|}{20.4} & \multicolumn{2}{|c|}{20.7} & \multicolumn{2}{|c|}{24.7} & \multicolumn{2}{|c|}{32.7} \\
\hline Implantation rate $(\%)$ & \multicolumn{2}{|c|}{29.5} & \multicolumn{2}{|c|}{34.9} & \multicolumn{2}{|c|}{26.7} & \multicolumn{2}{|c|}{28.9} & \multicolumn{2}{|c|}{10.7} \\
\hline Clinical pegnancy rate $(\%)$ & \multicolumn{2}{|c|}{38.7} & \multicolumn{2}{|c|}{45.6} & \multicolumn{2}{|c|}{35.1} & \multicolumn{2}{|c|}{24.7} & \multicolumn{2}{|c|}{14.0} \\
\hline Miscarriage rate $(\%)$ & \multicolumn{2}{|c|}{13.6} & \multicolumn{2}{|c|}{11.0} & \multicolumn{2}{|c|}{16.5} & \multicolumn{2}{|c|}{17.9} & \multicolumn{2}{|c|}{28.6} \\
\hline
\end{tabular}

Note: SD: standard deviation.

Table 3. Linear regression models of the effect of FSH dose on COS outcomes, at different maternal age intervals

\begin{tabular}{|c|c|c|c|c|c|c|c|c|}
\hline \multirow{2}{*}{$\begin{array}{l}\text { Group } \\
\text { Variable }\end{array}$} & \multicolumn{2}{|c|}{$\leq 35$ years old } & \multicolumn{2}{|c|}{$36-38$ years old } & \multicolumn{2}{|c|}{$39-40$ years old } & \multicolumn{2}{|c|}{$>40$ years old } \\
\hline & RC & $\boldsymbol{p}$ & RC & $\boldsymbol{p}$ & RC & $\boldsymbol{p}$ & RC & $\boldsymbol{p}$ \\
\hline \multicolumn{9}{|l|}{ cos outcomes } \\
\hline Aspirated follicles & -0.004 & NS & -0.003 & NS. & 0.000 & NS & 0.001 & NS \\
\hline Retrieved oocytes & -0.002 & NS & -0.001 & NS & 0.000 & NS & 0.000 & NS \\
\hline Oocyte Yield & 0.003 & 0.002 & 0.003 & NS & 0.003 & NS & 0.000 & NS \\
\hline Mature oocytes MII & -0.002 & NS & -0.001 & NS & 0.000 & NS & 0.000 & NS \\
\hline Mature oocytes rate & -0.001 & NS & -0.002 & NS & 0.002 & NS & -0.001 & NS \\
\hline
\end{tabular}

Note: COS: controlled ovarian stimulation; RC: regression coefficient, NS: non-significant $(p>0.05)$.

Table 4. Linear regression models of the effect of FSH dose on laboratorial outcomes, at different maternal age intervals.

\begin{tabular}{|c|c|c|c|c|c|c|c|c|}
\hline \multirow{2}{*}{$\frac{\text { Group }}{\text { Variable }}$} & \multicolumn{2}{|c|}{$\leq 35$ years old } & \multicolumn{2}{|c|}{$36-38$ years old } & \multicolumn{2}{|c|}{$39-40$ years old } & \multicolumn{2}{|c|}{$>40$ years old } \\
\hline & RC & $\boldsymbol{p}$ & RC & $\boldsymbol{p}$ & RC & $\boldsymbol{p}$ & RC & $\boldsymbol{p}$ \\
\hline \multicolumn{9}{|l|}{ Laboratory outcomes } \\
\hline Fertilization rate & -0.001 & NS & 0.000 & NS & 0.001 & NS & -0.002 & NS \\
\hline High quality D3 embryos & -0.001 & NS & 0.000 & NS & 0.000 & NS & 0.000 & NS \\
\hline High quality Embryo rate & -0.005 & $<0.01$ & -0.006 & 0.025 & 0.002 & NS & -0.002 & NS \\
\hline Blastocyst formation rate & -0.005 & $<0.01$ & -0.007 & 0.042 & 0.004 & NS & -0.004 & NS \\
\hline Transferred embryos & 0.000 & $<0.01$ & 0.000 & NS & 0.000 & NS & 0.000 & NS \\
\hline Endometrium & 0.000 & NS & 0.000 & NS & 0.001 & NS & 0.000 & NS \\
\hline
\end{tabular}

Note: RC: regression coefficient, NS: non-significant $(p>0.05)$.

Our evidence also showed that, especially in this group of women, the risk of OHSS is even higher. A gold standard COS should result in a sufficient number of retrieved oocytes, deviating from both undesirable results, OHSS and poor response. The question that rises is: "Should mild stimulation be applied for everyone?"

This could be of concern to older patients. Although the cumulative pregnancy rate (from fresh and frozen transfers from a single cycle, or from cumulative IVF cycles) was shown to be comparable with both approaches (Heijnen et al., 2007; Fatemi et al., 2013), this may not be a reality for everyone. Indeed, the development of less costly and more comfortable and practical protocols for COS is of pivotal importance, however, in developing countries, in which IVF treatments do not qualify for reimbursements, these protocols may not be a suitable alternative, and sometimes a single treatment may be the only option for these patients. 
Table 5. Linear regression models of the effect of clinical outcomes, at different maternal age intervals.

\begin{tabular}{|c|c|c|c|c|c|c|c|c|}
\hline \multirow{2}{*}{$\begin{array}{l}\text { Group } \\
\text { Variable }\end{array}$} & \multicolumn{2}{|c|}{$\leq 35$ years old } & \multicolumn{2}{|c|}{$36-38$ years old } & \multicolumn{2}{|c|}{$39-40$ years old } & \multicolumn{2}{|c|}{$>40$ years old } \\
\hline & RC & $\boldsymbol{p}$ & RC & $\boldsymbol{p}$ & $\mathbf{R C}$ & $\boldsymbol{p}$ & RC & $\boldsymbol{p}$ \\
\hline \multicolumn{9}{|l|}{ Clinical outcomes } \\
\hline Cancelation rate & 0.000 & 0.022 & 0.000 & 0.039 & 0.000 & NS & 0.000 & NS \\
\hline Implantation rate & -0.002 & NS & -0.004 & NS & -0.001 & NS & 0.001 & NS \\
\hline Clinical pegnancy rate & 0.000 & NS & 0.000 & NS & 0.000 & NS & 0.000 & NS \\
\hline Miscarriage rate & 0.001 & NS & 0.002 & NS & 0.001 & NS & 0.003 & NS \\
\hline
\end{tabular}

Note: RC: regression coefficient, NS: non-significant $(p>0.05)$.

\begin{tabular}{|l|c|c|c|c|c|c|c|c|c|}
\hline Table 6. One-way & ANOVA of the effect of age groups on FSH per oocyte ratio \\
\hline Group & $\mathbf{5 3}$ years old & $\mathbf{3 6 - 3 8}$ years old & $\mathbf{3 9 - 4 0}$ years old & \multicolumn{2}{|c|}{$>\mathbf{4 0}$ years old } & SD & Mean & SD & $\boldsymbol{p}$ \\
\hline Variable & Mean & SD & Mean & SD & Mean & SD \\
\hline FSH/oocyte ratio & 248.99 & 334.72 & 456.59 & 590.53 & 552.59 & 662.94 & 694.59 & 725.88 & $<0.05$ \\
\hline
\end{tabular}

Note: FSH: follicle-stimulated hormone; SD: standard deviation.

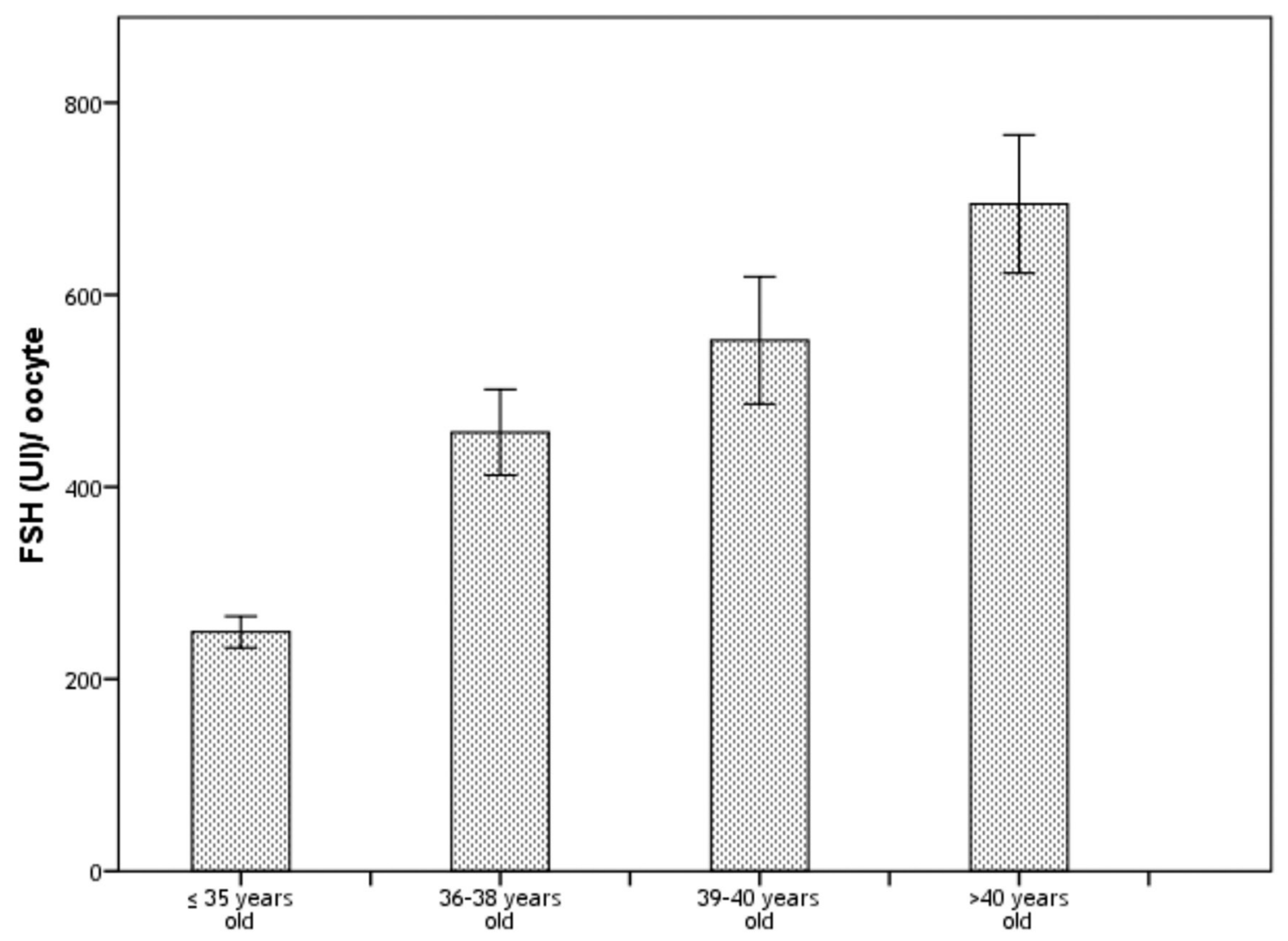

Figure 1. FSH per oocyte ratio. The mean FSH needed for one oocyte retrievel is represented in the bars. The error bars represent $95 \% \mathrm{CI}$. 
Our results suggested that in older patients, embryo quality and the blastocyst formation rates was not compromised by increased FSH doses. It could be argued that with advanced age, in general, the embryo quality is already impaired, and probably if there was a negative effect of FSH in the embryo biology, this was disguised in this group. On the other hand, to start the COS with lower FSH doses in older patients may not be an alternative, since it may lead to an increased rate of embryo transfer cancellation due to an insufficient number of oocytes retrieved.

As demonstrated here, the efficiency, in terms of FSH dose per retrieved oocyte, is much better in young patients. Then, mild stimulation protocols may be an alternative for younger women, since although higher FSH doses may result in increased oocyte yield, it significantly impacts the embryo developmental potential.

Lower FSH doses seem to be a great alternative to decrease discomfort, costs, and stress. Moreover, a mild ovarian response may be more appropriate for oocyte biology and it also works better in modern IVF protocols. With this approach, decreased number of embryos transferred and undesirable multiple pregnancies may be achieved, in addition, it results in less surplus embryos, and the inconvenience that it may cause, for storage. However, it may not be applicable for all patients, especially patients in advanced age.

In conclusion, mild ovarian stimulation protocols may be more appropriate for younger women ( $\leq 38$ years old), since our results indicated that, in these patients, there is a decrease in embryo quality and an increase in cycle's cancelation rate with the increase of FSH dose. However, this approach may not be useful for older woman $(\geq 38$ years old), given that higher FSH doses are needed to induce an adequate ovarian response, and this increase was not associated with impaired embryo development.

\section{CONFLICT OF INTEREST}

No conflict of interest has been declared.

\section{Corresponding author:}

Edson Borges Jr.

Fertility - Medical Group

São Paulo, SP - Brazil

E-mail: edson@fertility.com.br

\section{REFERENCES}

Aanesen A, Nygren KG, Nylund L. Modified natural cycle IVF and mild IVF: a 10 year Swedish experience. Reprod Biomed Online. 2010;20:156-62. PMID: 20159002 DOI: 10.1016/j.rbmo.2009.10.017

Ata B, Kaplan B, Danzer H, Glassner M, Opsahl M, Tan SL, Munné S. Array $\mathrm{CGH}$ analysis shows that aneuploidy is not related to the number of embryos generated. Reprod Biomed Online. 2012;24:614-20. PMID: 22503277 DOI: 10.1016/j.rbmo.2012.02.009

Baart EB, Martini E, Eijkemans MJ, Van Opstal D, Beckers NG, Verhoeff A, Macklon NS, Fauser BC. Milder ovarian stimulation for in-vitro fertilization reduces aneuploidy in the human preimplantation embryo: a randomized controlled trial. Hum Reprod. 2007;22:980-8. PMID: 17204525 DOI: $10.1093 /$ humrep/del484

Bodri D, Guillen JJ, Polo A, Trullenque M, Esteve C, Coll O. Complications related to ovarian stimulation and oocyte retrieval in 4052 oocyte donor cycles. Reprod Biomed Online. 2008;17:237-43. PMID: 18681998 DOI: $10.1016 / S 1472-6483(10) 60200-3$
Bosch E, Ezcurra D. Individualised controlled ovarian stimulation (iCOS): maximising success rates for assisted reproductive technology patients. Reprod Biol Endocrinol. 2011;9:82. PMID: 21693025 DOI: 10.1186/1477-7827-9-82

Braga DP, Setti AS, Figueira RC, Iaconelli A Jr, Borges E Jr. Contributing factors for the incidence of aneuploidy in older patients undergoing intracytoplasmic sperm injection cycles. J Assist Reprod Genet. 2012;29:911-6. PMID: 22644632 DOI: 10.1007/s10815-012-9795-x

Devroey P, Bourgain C, Macklon NS, Fauser BC. Reproductive biology and IVF: ovarian stimulation and endometrial receptivity. Trends Endocrinol Metab. 2004;15:84-90. PMID: 15036255 DOI: 10.1016/j.tem.2004.01.009

Dominguez F, Pellicer A, Simon C. The chemokine connection: hormonal and embryonic regulation at the human maternalembryonic interface-a review. Placenta. 2003;24:S48-55. PMID: 14559030 DOI: 10.1016/S0143-4004(03)00134-6

Fatemi HM, Blockeel C, Devroey P. Ovarian stimulation: today and tomorrow. Curr Pharm Biotechnol. 2012;13:3927. PMID: 21657998 DOI: $10.2174 / 138920112799362007$

Fatemi HM, Doody K, Griesinger G, Witjes H, Mannaerts B. High ovarian response does not jeopardize ongoing pregnancy rates and increases cumulative pregnancy rates in a GnRH-antagonist protocol. Hum Reprod. 2013;28:44252. PMID: 23136144 DOI: 10.1093/humrep/des389

Fauser BC, Devroey P, Macklon NS. Multiple birth resulting from ovarian stimulation for subfertility treatment. Lancet. 2005;365:1807-16. PMID: 15910954 DOI: $10.1016 / S 0140-6736(05) 66478-1$

Fauser BC, Nargund G, Andersen AN, Norman R, Tarlatzis B, Boivin J, Ledger W. Mild ovarian stimulation for IVF: 10 years later. Hum Reprod. 2010;25:2678-84. PMID: 20858698 DOI: $10.1093 /$ humrep/deq247

Goverde AJ, McDonnell J, Vermeiden JP, Schats R, Rutten FF, Schoemaker J. Intrauterine insemination or in-vitro fertilisation in idiopathic subfertility and male subfertility: a randomised trial and cost-effectiveness analysis. Lancet. 2000;355:13-8. PMID: 10615885 DOI: $10.1016 / S 0140-6736(99) 04002-7$

Haaf T, Hahn A, Lambrecht A, Grossmann B, Schwaab E, Khanaga O, Hahn T, Tresch A, Schorsch M. A high oocyte yield for intracytoplasmic sperm injection treatment is associated with an increased chromosome error rate. Fertil Steril. 2009;91:733-8. PMID: 18314124 DOI: $10.1016 /$ j.fertnstert.2008.01.012

Heijnen EM, Eijkemans MJ, De Klerk C, Polinder S, Beckers NG, Klinkert ER, Broekmans FJ, Passchier J, Te Velde ER, Macklon NS, Fauser BC. A mild treatment strategy for in-vitro fertilisation: a randomised noninferiority trial. Lancet. 2007;369:743-9. PMID: 17336650 DOI: $10.1016 /$ S0140-6736(07)60360-2

Hohmann FP, Macklon NS, Fauser BC. A randomized comparison of two ovarian stimulation protocols with gonadotropin-releasing hormone $(\mathrm{GnRH})$ antagonist cotreatment for in vitro fertilization commencing recombinant follicle-stimulating hormone on cycle day 2 or 5 with the standard long GnRH agonist protocol. J Clin Endocrinol Metab. 2003;88:166-73. PMID: 12519847 DOI: $10.1210 /$ jc. $2002-020788$ 
Hunault CC, Eijkemans MJ, Pieters MH, te Velde ER, Habbema JD, Fauser BC, Macklon NS. A prediction model for selecting patients undergoing in vitro fertilization for elective single embryo transfer. Fertil Steril. 2002;77:725-32. PMID: 11937124 DOI: $10.1016 /$ S0015-0282(01)03243-5

Jenkins JM, Davies DW, Devonport H, Anthony FW, Gadd SC, Watson RH, Masson GM. Comparison of 'poor' responders with 'good' responders using a standard buserelin/human menopausal gonadotrophin regime for in-vitro fertilization. Hum Reprod. 1991;6:918-21. PMID: 1761658 DOI: 10.1093/oxfordjournals.humrep.a137459

Kok JD, Looman CW, Weima SM, te Velde ER. A high number of oocytes obtained after ovarian hyperstimulation for in vitro fertilization or intracytoplasmic sperm injection is not associated with decreased pregnancy outcome. Fertil Steril. 2006;85:918-24. PMID: 16580375 DOI: $10.1016 /$ j.fertnstert.2005.09.035

Labarta E, Bosch E, Alamá P, Rubio C, Rodrigo L, Pellicer A. Moderate ovarian stimulation does not increase the incidence of human embryo chromosomal abnormalities in in vitro fertilization cycles. J Clin Endocrinol Metab. 2012;97:E1987-94. PMID: 22865900 DOI: $10.1210 /$ jc. 2012-1738

Pandian Z, Bhattacharya S, Ozturk O, Serour G, Templeton A. Number of embryos for transfer following in-vitro fertilisation or intra-cytoplasmic sperm injection. Cochrane Database Syst Rev. 2009;(2):CD003416. PMID: 19370588 DOI: $10.1002 / 14651858 . C D 003416$.pub3

Patrizio P, Sakkas D. From oocyte to baby: a clinical evaluation of the biological efficiency of in vitro fertilization. Fertil Steril. 2009;91:1061-6. PMID: 18325517 DOI: 10.1016/j.fertnstert.2008.01.003

Polyzos NP, Blockeel C, Verpoest W, De Vos M, Stoop $D$, Vloeberghs $V$, Camus $M$, Devroey $P$, Tournaye $H$. Live birth rates following natural cycle IVF in women with poor ovarian response according to the Bologna criteria. Hum Reprod. 2012;27:3481-6. PMID: 22940767 DOI: $10.1093 /$ humrep/des318

Seli E, Vergouw CG, Morita H, Botros L, Roos P, Lambalk CB, Yamashita N, Kato O, Sakkas D. Noninvasive metabolomic profiling as an adjunct to morphology for noninvasive embryo assessment in women undergoing single embryo transfer. Fertil Steril. 2010;94:535-42. PMID: 19589524 DOI: $10.1016 /$ j.fertnstert.2009.03.078

Setti PE, Bulletti C. Strategies to improve embryo implantation to supraphysiological rates. Ann N $Y$ Acad Sci. 2011;1221:75-9. PMID: 21401633 DOI: $10.1111 / j .1749-6632.2011 .05950 . x$

Shapiro BS, Daneshmand ST, Garner FC, Aguirre M, Hudson C, Thomas S. Evidence of impaired endometrial receptivity after ovarian stimulation for in vitro fertilization: a prospective randomized trial comparing fresh and frozen-thawed embryo transfer in normal responders. Fertil Steril. 2011;96:344-8. PMID: 21737072 DOI: $10.1016 /$ j.fertnstert.2011.05.050
Shulman A, Ghetler Y, Fejgin M, Kaneti H, Weinstein S, Beyth Y, Ben-Nun I. Relationship between the threshold of ovarian sensitivity to human menopausal gonadotrophin stimulation and in-vitro fertilization treatment outcome. Hum Reprod. 1995;10:3198-201. PMID: 8822443 DOI: 10.1093/oxfordjournals.humrep.a135887

Stadtmauer L, Ditkoff EC, Session D, Kelly A. High dosages of gonadotropins are associated with poor pregnancy outcomes after in vitro fertilization-embryo transfer. Fertil Steril. 1994;61:1058-64. PMID: 8194617 DOI: $10.1016 / S 0015-0282(16) 56756-9$

Steptoe PC, Edwards RG. Birth after the reimplantation of a human embryo. Lancet. 1978;2:366. PMID: 79723 DOI: $10.1016 /$ S0140-6736(78)92957-4

Steward RG, Lan L, Shah AA, Yeh JS, Price TM, Goldfarb JM, Muasher SJ. Oocyte number as a predictor for ovarian hyperstimulation syndrome and live birth: an analysis of 256,381 in vitro fertilization cycles. Fertil Steril. 2014;101:967-73. PMID: 24462057 DOI: $10.1016 /$ j.fertnstert.2013.12.026

Sunkara SK, Rittenberg V, Raine-Fenning N, Bhattacharya S, Zamora J, Coomarasamy A. Association between the number of eggs and live birth in IVF treatment: an analysis of 400135 treatment cycles. Hum Reprod. 2011;26:176874. PMID: 21558332 DOI: 10.1093/humrep/der106

Sunkara SK, La Marca A, Seed PT, Khalaf Y. Increased risk of preterm birth and low birthweight with very high number of oocytes following IVF: an analysis of 65868 singleton live birth outcomes. Hum Reprod. 2015;30:1473-80. PMID: 25883033 DOI: 10.1093/humrep/dev076

Sunkara SK, La Marca A, Polyzos NP, Seed PT, Khalaf Y. Live birth and perinatal outcomes following stimulated and unstimulated IVF: analysis of over two decades of a nationwide data. Hum Reprod. 2016;31:2261-7. PMID: 27591229 DOI: 10.1093/humrep/dew184

van der Gaast $\mathrm{MH}$, Eijkemans MJ, van der Net JB, de Boer EJ, Burger CW, van Leeuwen FE, Fauser BC, Macklon NS. Optimum number of oocytes for a successful first IVF treatment cycle. Reprod Biomed Online. 2006;13:476-80. PMID: 17007663 DOI: 10.1016/S1472-6483(10)60633-5

Verberg MF, Eijkemans MJ, Heijnen EM, Broekmans FJ, de Klerk C, Fauser BC, Macklon NS. Why do couples drop-out from IVF treatment? A prospective cohort study. Hum Reprod. 2008;23:2050-5. PMID: 18544578 DOI: $10.1093 /$ humrep/den219

Ziebe S, Lundin K, Janssens R, Helmgaard L, Arce JC; MERIT (Menotrophin vs Recombinant FSH in vitro Fertilisation Trial) Group. Influence of ovarian stimulation with HPhMG or recombinant FSH on embryo quality parameters in patients undergoing IVF. Hum Reprod. 2007;22:2404-13. PMID: 17640944 DOI: 10.1093/humrep/dem221 